\title{
Baryogenesis and gravitino dark matter in gauge-mediated supersymmetry-breaking models
}

\author{
Masaaki Fujii and T. Yanagida* \\ CERN Theory Division, CH-1211 Geneva 23, Switzerland \\ and Department of Physics, University of Tokyo, Tokyo 113-0033, Japan
}

(Received 2 August 2002; published 31 December 2002)

\begin{abstract}
We discuss two cosmological issues in a generic gauge-mediated supersymmetry- (SUSY-)breaking model, namely, the Universe's baryon asymmetry and the gravitino dark-matter density. We show that both problems can be simultaneously solved if there exist extra matter multiplets of a SUSY-invariant mass of the order of the " $\mu$ term," as suggested in several realistic SUSY grand-unified theories. We propose an attractive scenario in which the observed baryon asymmetry is produced in a way totally independent of the reheating temperature of inflation without causing any cosmological gravitino problem. Furthermore, in a relatively wide parameter space, we can also explain the present mass density of cold dark matter by the thermal relics of the gravitinos without an adjustment of the reheating temperature of inflation. We point out that there is an interesting relation between the baryon asymmetry and the dark-matter density.
\end{abstract}

DOI: 10.1103/PhysRevD.66.123515

PACS number(s): 98.80.Cq, 12.60.Jv, 98.80.Ft

\section{INTRODUCTION}

The mediation of supersymmetry- (SUSY-)breaking effects to the standard-model (SM) sector is a fundamental ingredient in the SUSY extension of the SM. Among various models proposed so far, the gauge mediation model with dynamical SUSY breaking (GMSB) [1] is the most attractive. It may not only solve the flavor-changing neutralcurrent (FCNC) problem, but also provide a natural explanation of the large hierarchy between the electroweak scale and the Planck scale because of the dynamical nature of SUSY breaking. Extensive analyses of the GMSB models have been performed on the physics at the $\mathrm{TeV}$ scale.

The remaining important task is to construct a successful cosmology in the GMSB models. The biggest difficulty comes from a stringent upper bound on the reheating temperature of inflation $T_{R}$ in order to avoid the overproduction of gravitinos [2]. The upper bound on $T_{R}$ is about $10^{6} \mathrm{GeV}$ when $m_{3 / 2} \simeq 10 \mathrm{MeV}$ for instance, and it even reaches $T_{R}$ $\$ 10^{3} \mathrm{GeV}$ in the case of the light gravitino $m_{3 / 2}$ $\$ 100 \mathrm{keV}$. In addition, we have to adjust the reheating temperature to just below this upper bound if we want to explain the required mass density of cold dark matter (CDM) by the gravitino LSPs (lightest SUSY particles). Furthermore, we also have to explain the required baryon asymmetry with just the same reheating temperature. These facts severely constrain models of inflation and the baryogenesis mechanism, and require a doubtful conspiracy between these two independent processes.

Even only explaining the required baryon asymmetry is very difficult. The unique mechanism to generate the baryon asymmetry in such low reheating temperatures, $T_{R}$ $\$ 10^{6} \mathrm{GeV}$, has been considered to be the Affleck-Dine (AD) baryogenesis [3]. ${ }^{1}$ However, in recent developments, it

\footnotetext{
* On leave from the University of Tokyo.

${ }^{1}$ In the case of a heavier gravitino $m_{3 / 2} \gtrsim 10 \mathrm{MeV}$, the leptogenesis through inflaton decays [4] or a coherently oscillating righthanded sneutrino [5] can explain the observed baryon asymmetry.
}

becomes clear that the coherent oscillation of the flat direction field (AD field) is unstable against spatial perturbations and that this field fragments into nontopological solitons called Q-balls [6] after dozens of its oscillations. In GMSB models, it is known that the resultant Q-balls are absolutely stable, and this fact has motivated some works to relate the observed baryon asymmetry and the mass density of dark matter [7]. Now that some detailed lattice simulations [8] have been performed, we can easily calculate the typical size and the number density of the Q-balls generated in GMSB models. Unfortunately, these simulations have revealed that the produced Q-balls generally overclose the Universe, which means that the usual AD baryogenesis is not a phenomenologically viable scenario as the origin of the observed baryon asymmetry in the GMSB models.

A possible solution to the difficulty is to adopt the $\mathrm{AD}$ field, which has a SUSY-invariant mass term. This is the case for the AD leptogenesis via the $L H_{u}$ flat direction [9]. The scenario can explain the baryon asymmetry provided that the " $\mu$ term" exists even in the very high energy (above the intermediate) scale, and that the mass of the lightest neutrino is extremely small [10]. ${ }^{2}$ However, we need a conspiracy again between the model of inflation and the leptogenesis if we want to explain the gravitino dark matter, simultaneously.

In this paper, we propose a solution to both problems. We consider the cosmology in GMSB models under the existence of extra matter multiplets of a SUSY-invariant mass of the order of the $\mu$ term. The existence of extra matter multiplets, such as those transforming $\mathbf{5}+\overline{\mathbf{5}}$ under the $\mathrm{SU}(5)_{\mathrm{GUT}}$ group, is indicated by several realistic SUSY grand unified theory (GUT) models [12] in which we can naturally solve the doublet-triplet splitting problem by utilizing some discrete symmetries.

As suggested from the $\mathrm{AD}$ leptogenesis, adopting an extra matter field as a member of the AD fields opens up various

\footnotetext{
${ }^{2}$ This fact allows us to have definite predictions on the rate of neutrinoless double beta $(0 \nu \beta \beta)$ decay, which will be tested in future experiments [11].
} 
possibilities to construct successful AD baryogenesis scenarios. In the present work, we will propose the most attractive one, which can generate the required baryon asymmetry in a way totally independent of the reheating temperature of inflation, without any conflicts with the constraint on cosmological gravitino abundance. Surprisingly, we can explain simultaneously, in a relatively wide parameter space, the required mass density of CDM by the thermal relics of the gravitino LSPs without an adjustment of the reheating temperature of inflation. In this region, we derive a novel relation between the mass density of the baryon and that of dark matter, which is written solely by low-energy parameters. We will also discuss the nonthermal gravitino production via late-time decays of the unstable Q-balls, and its phenomenological implications and experimental constraints.

\section{THE MODEL}

In the present scenario, we assume the existence of a pair of extra matter multiplets, $\mathbf{5}_{x}$ and $\overline{\mathbf{5}}_{x}$, which have a SUSYinvariant mass term in the superpotential, $W \supset m_{x} \mathbf{5}_{x} \overline{\mathbf{5}}_{x} \cdot{ }^{3}$ In the following discussion, we assume that the size of $m_{x}$ is of the order of the $\mu$ term $(|\mu| \sim 1 \mathrm{TeV})$, as suggested in realistic SUSY GUT models [12].

By adopting one of these extra fields as an AD field, this mass term supplies a quadratic potential along the relevant flat direction, which forbids the formation of stable Q-balls in the GMSB models. This is not the case, however, when the mass term is generated by an expectation value of some field with a renormalizable coupling to the extra matter multiplets, such as $W \supset S \mathbf{5}_{x} \overline{\mathbf{5}}_{x}$, where the $S$ field is to obtain an expectation value. If this is the case, the $S$ field is driven to the origin when the $\mathrm{AD}$ field develops a large amplitude, and then the required quadratic potential vanishes.

In order to avoid the formation of stable Q-balls in the GMSB models, the mass term should be present even after the AD field obtains a large amplitude. This condition can be easily satisfied, for example, if the mass term is generated by some nonrenormalizable operator [13], $W \supset\left(S^{2} / M_{*}\right) \mathbf{5}_{x} \overline{\mathbf{5}}_{x}$, where $M_{*} \equiv 2.4 \times 10^{18} \mathrm{GeV}$ is the reduced Planck scale. We assume that this is the case in the remainder of this paper.

Our model of the AD baryogenesis is basically the same as the one proposed in Sec. II B of Ref. [14]. We adopt the flat direction labeled by a linear combination of the monomials of the chiral superfields: $\bar{U} \bar{D} \bar{D}_{x}, Q \bar{D}_{x} L$. Here, $Q, \bar{U}, \bar{D}$ and $L$ denote superfields (and their scalar components) of a left-handed quark doublet, right-handed up- and down-type quarks and a left-handed lepton doublet, respectively; $\bar{D}_{x}$ belongs to the $\overline{\mathbf{5}}_{x}$ multiplet and has the same gauge quantum numbers as $\bar{D}$. As for an explicit parametrization of the flat direction, see Ref. [15].

The relevant baryon-number $(B)$ violating operators are

\footnotetext{
${ }^{3}$ The following arguments can also be applied to the case where the extra matter fields belong to $\mathbf{1 0}+\overline{\mathbf{1 0}}$ representations of the $\mathrm{SU}(5)_{\mathrm{GUT}}$.
}

supplied in the Kähler potential. (The effects of possible nonrenormalizable operators in the superpotential will be discussed later.) We take the following operators, for example, which are consistent with the $R$-symmetry:

$$
\delta K=\lambda_{1} \frac{1}{M_{*}^{2}} Q \bar{U}^{\dagger} \bar{D}_{x}^{\dagger} L+\lambda_{2} \frac{1}{M_{*}^{2}} Q \bar{U}^{\dagger} \bar{D}^{\dagger} L+\text { H.c. }
$$

where $\lambda_{i}$ 's are coupling constants. The SUSY-breaking effect due to the nonzero energy density of the Universe induces the following term in the scalar potential, which provides the $\mathrm{AD}$ field with a phase rotational motion:

$$
\delta V=3 H^{2} \delta K
$$

where $H$ is the Hubble parameter of the expanding Universe. Here, the fields in $\delta K$ represent the scalar components of corresponding superfields. ${ }^{4}$

The total scalar potential of the AD field $\phi$ can be written as follows:

$$
V=\left(m_{x}^{2}-c_{H} H^{2}\right)|\phi|^{2}+\frac{H^{2}}{4 M_{*}^{2}}\left(a_{H} \phi^{4}+\text { H.c. }\right)+\ldots,
$$

where the ellipsis denotes the higher-order terms coming from the Kähler potential; $a_{H}$ is a complex coupling constant and $c_{H}=\mathcal{O}(1)$ is a real positive coefficient. The definition of $\phi$ is the same as that in Ref. [15], which denotes the complex scalar field along the flat direction. The second term is induced from the four-point couplings between the inflaton $I$ and the $\mathrm{AD}$ field in the Kähler potential $\left(K \supset I^{\dagger} I \phi^{\dagger} \phi / M_{*}^{2}\right)$ [17].

\section{BARYON ASYMMETRY}

The evolution of the AD field and the resultant baryon asymmetry are almost the same as those presented in Refs. $[14,15]$. Here, we briefly review the important points. The AD field is assumed to develop a large expectation value during inflation, because of the large negative Hubbleinduced mass term. After the inflation ends, the Hubble parameter gradually decreases. The $\mathrm{AD}$ field starts coherent oscillations when $m_{x}$ eventually exceeds the Hubble param-

\footnotetext{
${ }^{4}$ The terms induced by the SUSY-breaking effect in the true vacuum have only a negligible role in the present scenario. Note that the existence of $\delta V$ in Eq. (2) is independent of the quantum charge of the inflaton. This is in clear contrast with the model proposed in Ref. [16], which requires the inflaton $I$ to be a singlet in order to write relevant interaction terms, $\delta K=\left(I / M_{*}\right) \phi^{\dagger} \phi$ and $\delta W=\left(I / M_{*}\right) \mathcal{W}_{\alpha} \mathcal{W}^{\alpha}$ to solve the Q-ball problem in the GMSB models. Such interactions generally lead to the high reheating temperature of inflation, which results in the overproduction of the gravitinos. In addition, there is no definite description of the available flat direction in the minimal supersymmetric standard model (MSSM) and the prediction of the resultant baryon asymmetry. It is not clear to us whether their scenario can actually produce the observed baryon asymmetry without a conflict with the cosmological gravitino problem.
} 
eter. At this time $H=H_{\mathrm{osc}} \simeq m_{x}$, a huge baryon asymmetry is generated by the $B$-violating operators in the scalar potential (3).

One can easily get the ratio of baryon to $\phi$-number density as

$$
\left(\frac{n_{B}}{n_{\phi}}\right) \simeq\left|a_{H}\right|\left(\frac{|\phi|_{0}}{M_{*}}\right)^{2} \delta_{\text {eff }}
$$

where $\delta_{\text {eff }} \equiv \sin \left[\arg \left(a_{H}\right)+4 \arg \left(\phi_{0}\right)\right]$. This is fixed at $H$ $=H_{\text {osc }}$ and remains constant until the $\phi$ fields decay into the gravitinos and SM particles. Here, $\phi_{0}$ denotes the expectation value of the $\mathrm{AD}$ field just after inflation. We treat it as a free parameter for a while, and assume that $|\phi|_{0} \lesssim M_{*}$. Actually, there exists a natural way to stop the $\mathrm{AD}$ field below the Planck scale. This can be done by gauging the $\mathrm{U}(1)_{B-L}$ symmetry. Although the $B$-violating operators in Eq. (1) are invariants of the $\mathrm{U}(1)_{B-L}$ symmetry, the relevant flat direction field $\phi$ carries nonzero $B-L$ charges and can be lifted by the $D$-term potential. In this case, the initial amplitude of the $\phi$ field is given by the $B-L$ breaking scale, $|\phi|_{0}$ $\simeq v_{B-L}[18]$.

In the present scenario, the quadratic potential supplied by the SUSY-invariant mass term of the extra matter field $\bar{D}_{x}$ forbids the formation of stable Q-balls. However, it cannot forbid the formation of unstable ones. This is because radiative corrections of $\mathrm{SU}(3)_{C}$ and $\mathrm{U}(1)_{Y}$ gauge interactions give rise to a negative contribution to the mass term $m_{x}^{2}|\phi|^{2}$. At the one-loop level, the potential is given by

$$
V=m_{x}^{2}\left[1+K \log \left(\frac{|\phi|^{2}}{M_{G}^{2}}\right)\right]|\phi|^{2},
$$

where

$$
K \simeq-\frac{1}{4 \pi}\left(\frac{16}{3} \alpha_{s}+\frac{4}{15} \alpha_{1}\right)
$$

Here, $M_{G}$ denotes a renormalization scale at which $m_{x}$ is defined. In this expression, we neglect the corrections from the possible Yukawa interactions, which should be small enough to avoid large FCNC interactions. In the following discussion, we use $K=-0.03$ for a representative value. ${ }^{5}$ These radiative corrections make the potential a little bit flatter than the quadratic one, which causes spatial instabilities and the formation of unstable Q-balls inevitable. A detailed discussion of the properties of the unstable Q-ball can be found in Ref. [14].

For the purpose of calculating the resultant baryon asymmetry, we need the decay temperature of the Q-ball. In the present model, the resultant Q-ball charge, $Q$, is estimated as

\footnotetext{
${ }^{5}$ It depends on the matter contents of the model and the scale of $|\phi|$. However, as we will see, the resultant baryon asymmetry and the mass density of dark matter depend only weakly on the precise value of the $K$-factor.
}

$$
Q=\bar{\beta}\left(\frac{|\phi|_{0}}{m_{x}}\right)^{2} \epsilon_{c}
$$

where $\beta \simeq 6 \times 10^{-3}$ and $\epsilon_{c} \simeq 0.01$ are determined by the detailed lattice simulations [8], which are independent of $|\phi|_{0}$. The size of the charge that evaporates from the surface of a single Q-ball through interactions with thermal backgrounds is about $\Delta Q \simeq 10^{18}[19]$. Consequently, the produced Q-balls survive thermal evaporation as long as $|\phi|_{0} \gtrsim 10^{14} \mathrm{GeV}$. The remaining charges are emitted through its decay into light fermions. The decay rate was calculated in Ref. [20] as

$$
\Gamma_{Q} \equiv-\frac{d Q}{d t} \lesssim \frac{\omega^{3} \mathcal{A}}{192 \pi^{2}} .
$$

Here, $\mathcal{A}=4 \pi R_{Q}^{2}$ is the surface area of the Q-ball, where $R_{Q} \simeq \sqrt{2} /\left(m_{x} \sqrt{|K|}\right)$ is the Q-ball radius; $\omega \simeq m_{x}$ is the effective mass of the Q-ball per $\phi$-number [21]. In the present model, the above inequality is almost saturated. A detailed discussion of the decay process and the condition for this saturation will be found in the next section.

From Eq. (8), the Q-ball decay temperature can be derived as follows:

$$
\begin{aligned}
T_{d} & =\frac{\eta}{\sqrt{48|K| \pi}}\left(\frac{90}{\pi^{2} g_{*}\left(T_{d}\right)}\right)^{1 / 4}\left(\frac{m_{x} M_{*}}{Q}\right)^{1 / 2} \\
& \simeq 2 \mathrm{GeV} \eta\left(\frac{0.03}{|K|}\right)^{1 / 2}\left(\frac{m_{x}}{1 \mathrm{TeV}}\right)^{1 / 2}\left(\frac{10^{20}}{Q}\right)^{1 / 2},
\end{aligned}
$$

where $\eta \sim 1$ denotes the ambiguity coming from an inequality of the decay rate in Eq. $(8) ; g_{*}\left(T_{d}\right)$ is the total number of effectively massless degrees of freedom at $T=T_{d}$. From Eq. (7), we can also write $T_{d}$ in terms of the initial amplitude of the $\phi$ field:

$$
\frac{T_{d}}{m_{x}}=\frac{\eta}{\sqrt{48 \pi|K| \bar{\beta} \epsilon_{c}}}\left(\frac{90}{\pi^{2} g_{*}\left(T_{d}\right)}\right)^{1 / 4}\left(\frac{m_{x} M_{*}}{|\phi|_{0}^{2}}\right)^{1 / 2} .
$$

The low decay temperature indicated in Eq. (9) makes it much easier for the Q-balls to dominate the energy density of the Universe. The condition for the Q-ball dominance is given by

$$
T_{R}>3 T_{d}\left(\frac{M_{*}}{|\phi|_{0}}\right)^{2}
$$

In the remainder of this paper, we consider the case where the above condition is satisfied. The corresponding region in the $\left(|\phi|_{0}-T_{R}\right)$ plane with thermal effects taken into account is presented in Ref. [15]. Basically, there is a wide parameter space for $|\phi|_{0} \geqslant 10^{15} \mathrm{GeV}$. In the case of $|\phi|_{0} \simeq M_{\mathrm{GUT}}$ $\left(\simeq 2 \times 10^{16} \mathrm{GeV}\right)$, for example, $10^{2} \mathrm{GeV} \leq T_{R} \lesssim 10^{10} \mathrm{GeV}$ is the viable range of the reheating temperature, which satisfies Eq. (11). Here, the upper bound on the reheating temperature comes from the condition to avoid the early oscillation of the AD field by the thermal effects [15]. 
In the case of the Q-ball dominance, the resultant baryon asymmetry is given by the following simple formula:

$$
\frac{n_{B}}{s}=\frac{\rho_{Q}}{s}\left(\frac{n_{\phi}}{\rho_{Q}}\right)\left(\frac{n_{B}}{n_{\phi}}\right)=\frac{3}{4} \frac{T_{d}}{m_{x}}\left|a_{H}\right|\left(\frac{|\phi|}{M_{*}}\right)^{2} \delta_{\mathrm{eff}},
$$

where $s, \rho_{Q}$ are the entropy density and the energy density of the Q-ball in the Universe, respectively. In terms of the density parameter of the baryon number, it is given by

$$
\begin{aligned}
\Omega_{B} h^{2} \simeq & 0.02 \times \eta\left(\frac{0.03}{|K|}\right)^{1 / 2}\left(\frac{m_{x}}{1 \mathrm{TeV}}\right)^{1 / 2} \\
& \times\left(\frac{|\phi|_{0}}{10^{16} \mathrm{GeV}}\right)\left(\frac{\left|a_{H}\right| \delta_{\text {eff }}}{0.02}\right),
\end{aligned}
$$

where $h$ is the present Hubble parameter in units of $100 \mathrm{~km} \mathrm{sec}^{-1} \mathrm{Mpc}^{-1}$, and $\Omega_{B} \equiv \rho_{B} / \rho_{c} ; \rho_{B}$ and $\rho_{c}$ are the energy density of the baryon and the critical density in the present Universe. Here, we have expressed the decay temperature $T_{d}$ of the Q-ball with the initial amplitude of the AD field and $m_{x}$ by using Eq. (10). The corresponding decay temperature of the Q-ball can be estimated as $T_{d}$ $\approx 200 \mathrm{MeV}$ for $|\phi|_{0} \approx 10^{16} \mathrm{GeV}$. Note that the resultant baryon asymmetry is totally independent of the reheating temperature of inflation and the detailed history of the Universe [14], since the final entropy density is solely produced via the late-time decays of Q-balls. The observed baryon asymmetry, $\Omega_{B} h^{2} \simeq 0.02$, suggests the initial amplitude of the AD field to be $10^{15} \mathrm{GeV} \lesssim|\phi|_{0} \lesssim 10^{17} \mathrm{GeV}$, which surprisingly coincides with the $B-L$ breaking scale suggested from the seesaw mechanism [22]. ${ }^{6}$

\section{GRAVITINO ABUNDANCE}

So far, we have concentrated on the calculation of the resultant baryon asymmetry in a Q-ball-dominated Universe. Now, let us turn our attention to the calculation of the relic gravitino abundance. In the present scenario, there are two stages in the gravitino production. The first one is the well known thermal gravitino production, which takes place at the reheating epoch of inflation. The second one is the nonthermal gravitino production from the late-time decays of the Q-balls.

\section{A. Thermal production}

First, let us estimate the abundance of thermal relics of gravitinos. It was calculated in detail in Ref. [2] and is given by

$$
\frac{n_{3 / 2}}{s}=\min \left\{\frac{45}{2 \pi^{2} g_{*}\left(T_{C}\right)} \frac{\zeta(3)}{\pi^{2}}\left(\frac{3}{2}\right), \frac{1}{4}\left(\frac{90}{\pi^{2} g_{*}\left(T_{R}\right)}\right)^{3 / 2}\left(\frac{\zeta(3)}{\pi^{2}}\right)^{2} T_{R} M_{*}\left\langle\Sigma_{\text {scatt }} v_{\text {rel }}\right\rangle\right\},
$$

where

$$
\left\langle\Sigma_{\text {scatt }} v_{\text {rel }}\right\rangle \approx 5.9 \frac{g_{3}^{2} m_{\widetilde{G}}^{2}}{m_{3 / 2}^{2} M_{*}^{2}}
$$

is the thermally averaged gravitino production cross section. Here, $m_{\tilde{G}}$ denotes the mass of the gluino. The first quantity in Eq. (14) denotes the gravitino abundance when it is thermalized; $T_{C}$ is the "decoupling" temperature of the gravitino, which is roughly given by

$$
\frac{\zeta(3)}{\pi^{2}} \frac{T_{C}^{3}\left\langle\Sigma_{\text {scatt }} v_{\text {rel }}\right\rangle}{H\left(T_{C}\right)}=\frac{3}{2}
$$

If the reheating temperature is higher than the mass of messenger (s)quarks $M_{m}$, gravitinos are easily thermalized. In this case, $T_{C}$ should be replaced by the smaller one between $M_{m}$ and the solution $T_{C}$ of Eq. (16).

Thermally produced gravitinos presented in Eq. (14) are significantly diluted by the subsequent entropy production associated with the Q-ball decays. The dilution factor via the late-time decays of Q-balls is given by [14]

$$
\frac{1}{\Delta}=3 \frac{T_{d}}{T_{\text {ini }}}\left(\frac{M_{*}}{|\phi|_{0}}\right)^{2}
$$

where

$$
T_{\mathrm{ini}}=\min \left[T_{R}, \sqrt{m_{x} M_{*}}\left(\frac{90}{\pi^{2} g_{*}}\right)^{1 / 4}\right]
$$

The second term in the square bracket of Eq. (18) denotes the case where the $\mathrm{AD}$ field starts coherent oscillation after the reheating process of inflation. In this case, it is difficult for the AD field with $|\phi|_{0} \simeq M_{G U T}$ to escape strong thermal effects, which modify the resultant baryon asymmetry and the properties of the produced Q-balls. Therefore, we concentrate on the other case, and assume that

$$
T_{R} \lesssim \sqrt{m_{x} M_{*}}\left(\frac{90}{\pi^{2} g_{*}}\right)^{1 / 4} \approx 2 \times 10^{10} \mathrm{GeV}
$$

\footnotetext{
${ }^{6}$ The mass of the right-handed neutrino $M_{R}$ is given by $M_{R}$ $=\lambda v_{B-L}$, where $\lambda$ is some coupling constant. This fact and the atmospheric-neutrino data naturally suggest $v_{B-L} \sim M_{\mathrm{GUT}}$.
} 
in the remainder of this paper.

First, let us consider the case where the gravitinos are thermalized. From Eqs. (14) and (17), we obtain

$$
\frac{n_{3 / 2}}{s}=1.25 \times \frac{T_{d}}{g_{*}\left(T_{C}\right) T_{R}}\left(\frac{M_{*}}{|\phi|_{0}}\right)^{2} .
$$

In terms of the density parameter, it becomes

$$
\begin{aligned}
\Omega_{3 / 2} h^{2} \simeq & 0.2 \times \eta\left(\frac{0.03}{|K|}\right)^{1 / 2}\left(\frac{300}{g_{*}\left(T_{C}\right)}\right)\left(\frac{m_{3 / 2}}{1 \mathrm{MeV}}\right) \\
& \times\left(\frac{10^{8} \mathrm{GeV}}{T_{R}}\right)\left(\frac{m_{x}}{1 \mathrm{TeV}}\right)^{3 / 2}\left(\frac{10^{16} \mathrm{GeV}}{|\phi|_{0}}\right)^{3} .
\end{aligned}
$$

Here, we have used the relation in Eq. (10). The required mass density of CDM, $\Omega_{3 / 2} h^{2} \simeq 0.1-0.2$, can be easily explained by adjusting the reheating temperature of inflation. Note that this adjustment does not change the resultant baryon asymmetry, since it is independent of the reheating temperature [see Eq. (13)].

A much more interesting situation arises if the gravitinos are not thermalized in the early Universe. If this is the case, from Eqs. (14) and (17), the resultant gravitino abundance is given by

$$
\frac{n_{3 / 2}}{s}=0.31 \times \frac{T_{d} M_{*}}{g_{*}\left(T_{R}\right)^{3 / 2}\left\langle\Sigma_{\text {scatt }} v_{\text {rel }}\right\rangle^{-1}}\left(\frac{M_{*}}{|\phi|_{0}}\right)^{2} .
$$

One can see that the resultant gravitino abundance is also independent of the reheating temperature of inflation. In terms of the density parameter, this is written as

$$
\begin{aligned}
\Omega_{3 / 2} h^{2} \simeq & 0.16 \times \eta\left(\frac{250}{g_{*}\left(T_{R}\right)}\right)^{3 / 2}\left(\frac{0.03}{|K|}\right)^{1 / 2}\left(\frac{1 \mathrm{MeV}}{m_{3 / 2}}\right) \\
& \times\left(\frac{m_{\tilde{G}}}{1 \mathrm{TeV}}\right)^{2}\left(\frac{m_{x}}{1 \mathrm{TeV}}\right)^{3 / 2}\left(\frac{M_{\mathrm{GUT}}}{|\phi|_{0}}\right)^{3} .
\end{aligned}
$$

Therefore, in this case, both the baryon asymmetry and the mass density of gravitino dark matter are solely determined by electroweak-scale parameters and by the initial amplitude of the AD field. Encouragingly, both quantities can be consistent with observations in a reasonable parameter space. ${ }^{7}$

In fact, we can derive a novel relation between the mass density of baryons and that of the gravitino dark matter in terms of low-energy parameters:

\footnotetext{
${ }^{7}$ Contributions to the mass density of dark matter from the nonthermal gravitino production should be subdominant. See the next subsection.
}

$$
\begin{aligned}
\left(\Omega_{B} h^{2}\right) & \times\left(\Omega_{3 / 2} h^{2}\right)^{1 / 3} \\
\simeq & 1.1 \times 10^{-2} \eta^{4 / 3}\left(\frac{250}{g_{*}\left(T_{R}\right)}\right)^{1 / 2}\left(\frac{1 \mathrm{MeV}}{m_{3 / 2}}\right)^{1 / 3} \\
& \times\left(\frac{m_{\widetilde{G}}}{1 \mathrm{TeV}}\right)^{2 / 3}\left(\frac{m_{x}}{1 \mathrm{TeV}}\right)\left(\frac{0.03}{|K|}\right)^{2 / 3}\left(\frac{\left|a_{H}\right| \delta_{\mathrm{eff}}}{0.01}\right),
\end{aligned}
$$

which is quite consistent with the observation. We should stress that the above combination of the baryon asymmetry and the gravitino dark-matter density is independent of the unknown high energy physics parameters, such as $|\phi|_{0}$ and $T_{R}$. There remain only a weak dependence on the effective degrees of freedom, $g_{*}\left(T_{R}\right)$, and a natural assumption on the coupling constant and the effective $C P$-violating phase, $\left|a_{H}\right| \delta_{\text {eff }}=\mathcal{O}(0.01-0.1)$.

We can obtain another interesting relation by taking a ratio of the mass density of a baryon to that of dark matter:

$$
\begin{aligned}
\frac{\Omega_{B}}{\Omega_{3 / 2}} \simeq & 0.2 \times\left(\frac{m_{3 / 2}}{1 \mathrm{MeV}}\right)\left(\frac{1 \mathrm{TeV}}{m_{x}}\right)\left(\frac{1 \mathrm{TeV}}{m_{\tilde{G}}}\right)^{2} \\
& \times\left(\frac{g_{*}\left(T_{R}\right)}{250}\right)^{3 / 2}\left(\frac{|\phi|_{0}}{M_{\mathrm{GUT}}}\right)^{4}\left(\frac{\left|a_{H}\right| \delta_{\mathrm{eff}}}{0.02}\right) .
\end{aligned}
$$

As one can see, if the $(B-L)$-breaking scale (and hence $\left.|\phi|_{0}\right)$ is around the GUT scale, the present scenario predicts a successful relation between baryon asymmetry and dark matter.

In Fig. 1, we show a parameter space where we can explain the gravitino CDM independently of the reheating temperature of inflation. [As we have mentioned before, if the reheating temperature is higher than the mass of the messenger (s)quarks, the gravitino abundance should be calculated by Eq. (21).]

Finally, we add a brief comment on the possible isocurvature perturbations. In the present scenario, the origin of the CDM is the inflaton decay, but the dominant entropy is produced by the late-time decays of Q-balls. Thus, the density fluctuation of the CDM contains partially isocurvature perturbations if the dominant-density perturbations are supplied by the fluctuation of the AD field during inflation [23]. In order to avoid any disagreement with observations, the Hubble parameter during inflation should satisfy $H_{I}$ $\lesssim 10^{-5}|\phi|_{0} \approx 10^{10-12} \mathrm{GeV} .^{8}$ However, this is not a strong constraint, and it can be easily satisfied, for example, in hybrid and new inflation models [24]. It will be an interesting indication of the present model, if a small deviation from the purely adiabatic density perturbations is indeed confirmed in the future experiments, such as Microwave Anisotropy Probe (MAP) [25] and Planck [26].

\footnotetext{
${ }^{8}$ In this case, the dominant-density perturbations are generated by the inflaton, and there remain small isocurvature perturbations in the baryonic sector.
} 


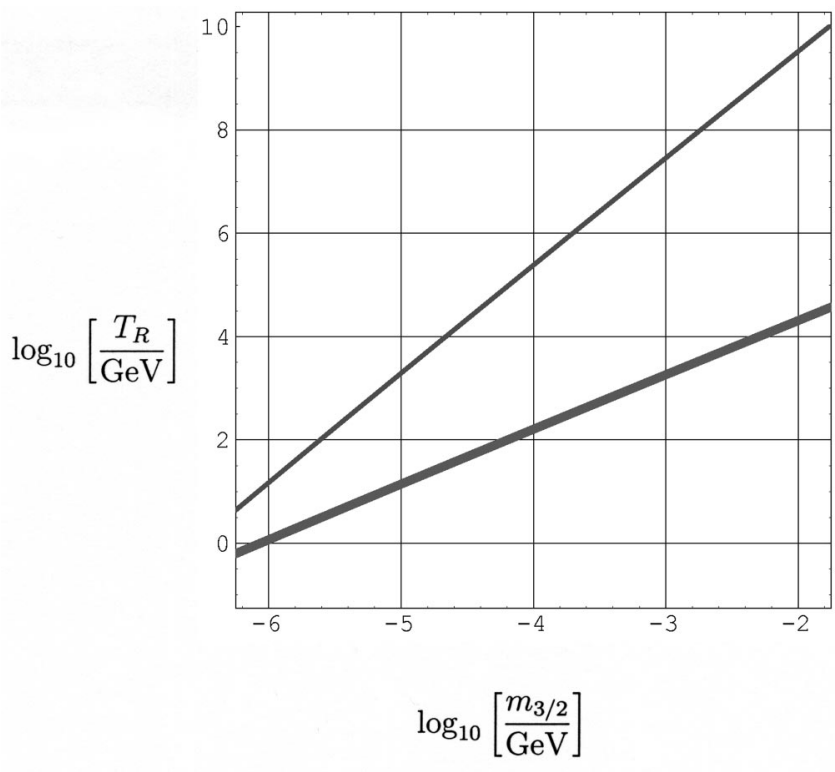

FIG. 1. The parameter space where the mass density of gravitino $\mathrm{CDM}$ is determined independently of the reheating temperature of inflation. Below the thick line, the mass density of the gravitino is too small to explain the required mass density, $\Omega_{3 / 2} h^{2}<0.05$. Above the thin line, the gravitinos are thermalized by scattering interactions. The region between the two lines, the required mass density of gravitino dark matter, can be explained by Eq. (23). Here, we have assumed that the reheating temperature $T_{R}$ is smaller than the messenger (s)quark masses.

\section{B. Nonthermal production}

In this subsection, we discuss the nonthermal gravitino production from the late-time decays of Q-balls. We first estimate the resultant nonthermal gravitino abundance, and then discuss experimental constraints and phenomenological implications.

As we have mentioned in Sec. III, the AD field stored inside the Q-ball decays into its superpartner and a gaugino, or a quark-lepton and a Higgsino, through gauge or Yukawa interactions, respectively. The following condition is necessary for the $\mathrm{AD}$ field to decay into light fermions with an almost saturated rate $(\eta \sim 1)$ in Eq. (8),

$$
f|\phi|_{c} \gtrsim \omega\left(\simeq m_{x}\right),
$$

where $f$ denotes the corresponding gauge or Yukawa coupling constant, and $|\phi|_{c}$ is the amplitude of the AD field at the center of the Q-ball. We can easily calculate the typical amplitude of the AD field inside the Q-ball from its charge and radius, which turns out to be $|\phi|_{c} \approx 10^{12} \mathrm{GeV}$ for $|\phi|_{0}$ $\simeq M_{\mathrm{GUT}}$ and clearly satisfies the above condition.

This is not the case for the decay of the AD field into the gravitino. The relevant interaction is given by

$$
\mathcal{L} \supset \frac{m_{\chi_{\phi}}^{2}-m_{\phi}^{2}}{\sqrt{3} m_{3 / 2} M_{*}}\left(\bar{\psi} \chi_{\phi}\right) \phi^{*}+\text { H.c. },
$$

where $\chi_{\phi}$ denotes the superpartner of the AD field $\phi$, and $\psi$ is the longitudinal component of the gravitino ( $\sim$ the Gold- stino). In the region where $|\phi| \geqslant M_{m}$, the mass difference of the AD field and its superpartner is given by [27]

$$
m_{\phi}^{2}-m_{\chi_{\phi}}^{2} \simeq m_{\mathrm{soft}}^{2} \frac{M_{m}^{2}}{|\phi|^{2}},
$$

where $m_{\text {soft }} \simeq 1 \mathrm{TeV}$ is the soft SUSY-breaking mass of the AD field below the messenger scale $M_{m}$. As a result, the branching ratio of the $\mathrm{AD}$ field that directly decays into the gravitino is suppressed by the following factor with respect the decay rate given in Eq. (8) [20]:

$$
\frac{m_{\text {soft }}^{2} M_{m}^{2} /|\phi|}{\sqrt{3} m_{3 / 2} M_{*}} \times \frac{1}{\omega} .
$$

Below the messenger scale $|\phi| \leq M_{m}$, the numerator in (29) is just given by $m_{\text {soft }}^{2}|\phi|$. Thus the branching ratio is maximized at $|\phi| \simeq M_{m}$. The resultant gravitino abundance directly generated from the Q-ball decay is given by

$$
\begin{aligned}
\Omega_{3 / 2}^{\text {direct }} h^{2} \approx & \left(\Omega_{B} h^{2}\right)\left(\frac{n_{\phi}}{n_{B}}\right) \times \frac{m_{\text {soft }}^{2} M_{m}}{\sqrt{3} M_{*} m_{x} m_{p}} \\
\simeq & 5 \times 10^{-6}\left(\frac{\left(n_{\phi} / n_{B}\right)}{10^{6}}\right)\left(\frac{m_{\text {soft }}}{1 \mathrm{TeV}}\right)^{2} \\
& \times\left(\frac{1 \mathrm{TeV}}{m_{x}}\right)\left(\frac{M_{m}}{10^{6} \mathrm{GeV}}\right),
\end{aligned}
$$

where $m_{p}$ is the mass of a nucleon. In order to explain the observed baryon asymmetry, the ratio of $n_{\phi}$ to $n_{B}$ is given by $\left(n_{\phi} / n_{B}\right) \approx 10^{6}$ from Eqs. (4) and (13). Therefore, from the above equation, the mass density of gravitinos directly produced by the decays of the AD fields inside the Q-balls is a negligible contribution to the total mass density of dark matter, as long as $M_{m} \leq 10^{10} \mathrm{GeV}$.

A superparticle produced by the late-time decays of Q-balls subsequently decays into the next-to-lightest supersymmetric particle (NLSP $\chi$ ), which is the $B$-ino or the lightest stau in the minimal GMSB models. The NLSP, in turn, decays into the gravitino and its superpartner with the following decay width:

$$
\Gamma_{\chi} \simeq \frac{1}{48 \pi} \frac{m_{\chi}^{5}}{m_{3 / 2}^{2} M_{*}^{2}},
$$

where $m_{\chi}$ is the mass of the NLSP. In order not to spoil the success of big bang nucleosynthesis (BBN), it must satisfy the following constraint [28]:

$$
\begin{aligned}
T_{3 / 2} & \equiv\left(\frac{90}{\pi^{2} g_{*}\left(T_{3 / 2}\right)}\right)^{1 / 4} \sqrt{\Gamma_{\chi} M_{*}} \\
& \simeq 5 \mathrm{MeV}\left(\frac{m_{\chi}}{100 \mathrm{GeV}}\right)^{5 / 2}\left(\frac{1 \mathrm{MeV}}{m_{3 / 2}}\right) \gtrsim 5 \mathrm{MeV} .
\end{aligned}
$$


This condition is satisfied for a gravitino with $m_{3 / 2} \lesssim$ (a few) $\mathrm{MeV}$ for a reasonable range of mass of $\chi$.

First, let us consider the case where

$$
H\left(T_{d}\right)\left(=\sqrt{\frac{\pi^{2} g_{*}\left(T_{d}\right)}{90}} \frac{T_{d}^{2}}{M_{*}}\right) \gg \Gamma_{\chi} .
$$

Here, the produced NLSPs have enough time to reduce their abundance via annihilations into the following value [29] before they decay into gravitinos:

$$
\frac{n_{\chi}}{s}=\sqrt{\frac{45}{8 \pi^{2} g_{*}\left(T_{d}\right)}} \frac{\langle\sigma v\rangle_{\chi}^{-1}}{M_{*} T_{d}},
$$

where $\langle\sigma v\rangle_{\chi}$ denotes the s-wave component of the annihilation cross section of the NLSP. As a result, the resultant gravitino abundance is given by

$$
\begin{aligned}
\Omega_{3 / 2}^{\chi} h^{2} \simeq & 2.8 \times 10^{-2}\left(\frac{m_{3 / 2}}{1 \mathrm{MeV}}\right)\left(\frac{10^{-11} \mathrm{GeV}^{-2}}{\langle\sigma v\rangle_{\chi}}\right) \\
& \times\left(\frac{100 \mathrm{MeV}}{T_{d}}\right) .
\end{aligned}
$$

In the case where $H\left(T_{d}\right) \lesssim \Gamma_{\chi}$, the estimation given in Eq. (35) is not valid, since the NLSPs produced by the Q-ball decays do not have enough time to annihilate before decaying into gravitinos. In this case, we have to solve the following coupled Boltzmann equations [14]:

$$
\begin{gathered}
\dot{n}_{\chi}+3 H n_{\chi}=N_{\chi} \Gamma_{Q} n_{Q}^{\text {total }}-\langle\sigma v\rangle_{\chi} n_{\chi}^{2}-\Gamma_{\chi} n_{\chi}, \\
n_{Q}^{\text {total }}+3 H n_{Q}^{\text {total }}=0 \quad\left(\text { for } t \leqslant \tau_{d}\right), \\
n_{Q}^{\text {total }}=0 \quad\left(\text { for } t \geqslant \tau_{d}\right), \\
H^{2}=\frac{1}{3 M_{*}^{2}}\left(\rho_{Q}+\rho_{\chi}+\rho_{\text {rad }}\right), \\
\rho_{Q}=\epsilon_{c}^{-1} m_{\phi}\left(Q_{i}-\Gamma_{Q} t\right) n_{Q}^{\text {total }}, \\
\rho_{\chi}=m_{\chi} n_{\chi}, \\
\dot{\rho}_{\text {rad }}+4 H \rho_{\text {rad }}=\left(\epsilon_{c}^{-1} m_{\phi}-N_{\chi} m_{\chi}\right) \Gamma_{Q} n_{Q}^{\text {total }} \\
+m_{\chi}\langle\sigma v\rangle_{\chi} n_{\chi}^{2}+m_{\chi} \Gamma_{\chi} n_{\chi}, \\
\dot{n}_{3 / 2}+3 H n_{3 / 2}=\Gamma_{\chi} n_{\chi},
\end{gathered}
$$

where $n_{Q}^{\text {total }}$ is the total number density of Q-balls, which includes positively and negatively charged Q-balls, ${ }^{9} m_{\phi}$

\footnotetext{
${ }^{9}$ As for the details, see Ref. [14] and references therein.
}

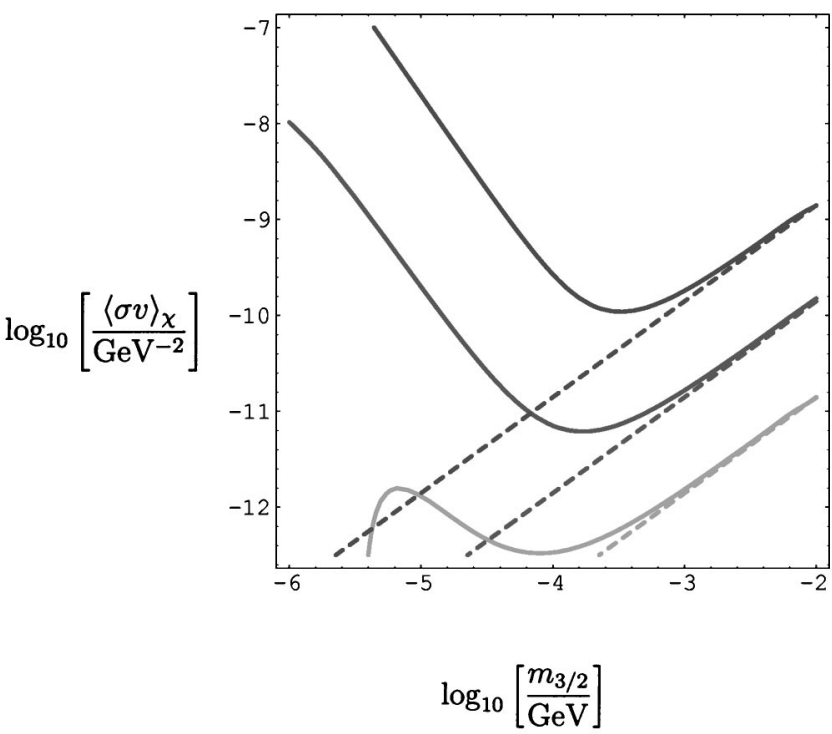

FIG. 2. The contour plot of the mass density of nonthermal gravitinos $\Omega_{3 / 2}^{\chi} h^{2}$ in the $\left(m_{3 / 2}-\langle\sigma v\rangle_{\chi}\right)$ plane. The solid lines correspond to $\Omega_{3 / 2}^{\chi} h^{2}=0.15,0.015$ and 0.0015 from the bottom up. The dashed lines represent the corresponding mass density of gravitinos calculated from Eq. (35).

$\simeq m_{x}$ is the mass of the AD field, $n_{3 / 2}$ is the number density of gravitinos, $Q_{i}$ is the initial charge of the Q-ball, $\tau_{d}$ $\equiv Q_{i} / \Gamma_{Q}$ is the lifetime of the Q-ball, and $N_{\chi}$ is the number of NLSPs produced per baryon number, which is roughly equal to $\epsilon_{c}^{-1}$. Note that the produced gravitinos behave as radiation for the era relevant to the present calculation. In Fig. 2, we show the contour plot of $\Omega_{3 / 2}^{\chi} h^{2}$ by numerically solving the above Boltzmann equations. Here, we have assumed that $T_{d}=100 \mathrm{MeV}, m_{x}=1 \mathrm{TeV}, m_{\chi}=100 \mathrm{GeV}$, $\epsilon_{c}^{-1}=N_{\chi}=100$.

Now, we discuss experimental constraints on the nonthermal gravitino production. First, let us consider the constraint from BBN. Even if the Q-balls and the NLSPs decay into gravitinos well before the start of the BBN [see Eq. (32)], the produced gravitinos are still ultrarelativistic during the era, which may reduce too much the energy density during the formation of light elements.

The momentum of the nonthermal gravitino redshifts with temperature, ${ }^{10}$

$$
p(T) \simeq \frac{m_{\chi}}{2}\left(\frac{g_{*}(T)}{g_{*}\left(T_{g}\right)}\right)^{1 / 3} \frac{T}{T_{g}},
$$

where $T_{g}$ is the cosmic temperature at the time of the gravitino production. Note that $T_{g}$ depends on the production mechanism and the relative size between $H\left(T_{d}\right)$ and $\Gamma_{\chi}$, the decay rate of the NLSP, and that $T_{g}$ is given by

\footnotetext{
${ }^{10}$ In the case of the direct production of gravitinos from the AD fields stored in the Q-balls, $m_{\chi}$ should be replaced by $m_{x}$.
} 


$$
\begin{gathered}
T_{g}=T_{3 / 2} \quad \text { for } \quad H\left(T_{d}\right) \gtrsim \Gamma_{\chi} \\
T_{g}=T_{d} \text { for } \quad H\left(T_{d}\right) \lesssim \Gamma_{\chi}
\end{gathered}
$$

and the direct gravitino production. Therefore, the contribution to the energy density of the radiation from the nonthermal gravitinos at temperature $T$ is estimated to

$$
\begin{aligned}
\rho_{3 / 2}(T) \simeq & \left(\frac{n_{3 / 2}}{s}\right) s(T) p(T) \\
\simeq & 7.8 \times 10^{-3}\left(\frac{g_{*}(T)^{\frac{4}{3}}}{g_{*}\left(T_{g}\right)^{1 / 3}}\right)\left(\frac{m_{\chi}}{100 \mathrm{GeV}}\right) \\
& \times\left(\frac{100 \mathrm{MeV}}{T_{g}}\right)\left(\frac{100 \mathrm{keV}}{m_{3 / 2}}\right)\left(\Omega_{3 / 2}^{\mathrm{NT}} h^{2}\right) T^{4},
\end{aligned}
$$

where $\Omega_{3 / 2}^{\mathrm{NT}} h^{2}$ denotes the present mass density of nonthermal gravitino dark matter, which is $\Omega_{3 / 2}^{\text {direct }} h^{2}$ or $\Omega_{3 / 2}^{\chi} h^{2}$.

In order not to affect the expansion of the Universe during $\mathrm{BBN}$, the gravitino contribution to the energy density of radiation should satisfy

$$
\frac{\rho_{3 / 2}}{\rho_{\nu}} \leqslant \delta N_{\nu}
$$

where $\rho_{\nu}=\left(\pi^{2} / 30\right)(7 / 4) T^{4}$ is the energy density of one neutrino species. Agreement with observations of light elements requires $\delta N_{\nu}=0.2-1$ [30]. This leads to the following constraint:

$$
\begin{aligned}
\frac{\rho_{3 / 2}}{\rho_{\nu}} \simeq & 1.4 \times 10^{-3}\left(\frac{g_{*}(T)^{4 / 3}}{g_{*}\left(T_{g}\right)^{1 / 3}}\right)\left(\frac{m_{\chi}}{100 \mathrm{GeV}}\right) \\
& \times\left(\frac{100 \mathrm{MeV}}{T_{g}}\right)\left(\frac{100 \mathrm{keV}}{m_{3 / 2}}\right)\left(\frac{\Omega_{3 / 2}^{\mathrm{NT}} h^{2}}{0.1}\right) \\
\leqslant & 0.2-1 .
\end{aligned}
$$

As can be seen, this constraint is very weak and almost always satisfied for the interested region of the parameter space.

Although the constraint coming from BBN is very weak, the nonthermal gravitinos should not make up a dominant portion of dark matter. This is because they maintain relativistic velocity until just before the matter-radiation equality. Actually, they are too hot to constitute acceptable warm dark matter, even though they are a little bit cooler than neutrinos. The current velocity of the nonthermal gravitino is given by $^{11}$

\footnotetext{
${ }^{11}$ In the case of a direct production, $m_{\chi}$ should be replaced by $m_{x}$.
}

$$
\begin{aligned}
v_{0} & \simeq \frac{m_{\chi}}{2 m_{3 / 2}}\left(\frac{g_{*}\left(T_{0}\right)}{g_{*}\left(T_{g}\right)}\right)^{1 / 3} \frac{T_{0}}{T_{g}} \\
& \simeq 1.8 \times 10^{-6}\left(\frac{1 \mathrm{MeV}}{m_{3 / 2}}\right)\left(\frac{m_{\chi}}{100 \mathrm{GeV}}\right)\left(\frac{5 \mathrm{MeV}}{T_{g}}\right),
\end{aligned}
$$

where $T_{0}=2.7 \mathrm{~K}$ is the current temperature of the cosmic microwave background (CMB). Note that the velocity $v_{0}$ is independent of $m_{3 / 2}$ as long as $H\left(T_{d}\right) \geqslant \Gamma_{\chi}$, since the gravitino mass dependence is cancelled out by the $m_{3 / 2}$ dependence of its decay temperature $T_{3 / 2}$. This is the case when $m_{3 / 2} \gtrsim 100 \mathrm{keV}$ for the typical Q-ball decay temperature $T_{d}$ $\approx 100 \mathrm{MeV}$. In the case of lighter gravitino $m_{3 / 2}$ $\lesssim 100 \mathrm{keV}, v_{0}$ is inversely proportional to the gravitino mass, since $T_{g}=T_{d} \approx 100 \mathrm{MeV}$ is independent of the gravitino mass [see Eq. (45)]. On the other hand, $v_{0}$ $\lesssim 1.5 \times 10^{-7}$ is required for warm dark matter to be consistent with observations of the Lyman- $\alpha$ forest [31,32], which corresponds to the free-streaming scale $R_{f} \lesssim 0.1 \mathrm{Mpc}$. In the present scenario, $R_{f} \approx 1 \mathrm{Mpc}$ for $m_{3 / 2} \gtrsim 100 \mathrm{keV}$, and it even reaches $R_{f} \approx 100 \mathrm{Mpc}$ for $m_{3 / 2} \approx 1 \mathrm{keV}$.

Therefore, the contribution from the nonthermally produced gravitinos should be a subdominant component of dark matter; otherwise it would be in conflict with the observed large-scale structure of the Universe. Although there has been derived no accurate bound on the mass density of such "hotter" warm dark matter, we can obtain a conservative bound by treating it as hot dark matter. CMB measurements and galaxy cluster surveys already constrain the mass density of hot dark matter as $\Omega_{\text {hot }} h^{2} \lesssim 0.05$ at the $95 \%$ C.L. [33]. From Eq. (30) and Fig. 2, one can see that this constraint is satisfied as long as $M_{m} \lesssim 10^{10} \mathrm{GeV}$ and $\langle\sigma v\rangle_{\chi}$ $\gtrsim($ a few $) \times 10^{-12} \mathrm{GeV}^{-2}$. In the case of the stau NLSP, the s-wave annihilation cross section is of the order of $10^{-7} \mathrm{GeV}^{-2}\left(100 \mathrm{GeV} / m_{\chi}\right)^{2}$, which clearly satisfies the condition. Even in the case of the $B$-ino NLSP, $\langle\sigma v\rangle_{\chi}$ $\gtrsim 10^{-11} \mathrm{GeV}^{-2}$ is naturally obtained for relatively large $\tan \beta$ ( $\gtrsim 15)$. If the nonthermal gravitinos make up at least a few percent of the total mass density of dark matter, data from the MAP or Planck satellites may confirm its existence in the near future [34].

\section{CONCLUSIONS AND DISCUSSION}

In this work, we have proposed a solution to the baryon asymmetry and the dark matter problems in the GMSB models. We have found that both problems can be simultaneously solved if there exist extra matter multiplets of a SUSYinvariant mass of the order of the $\mu$ term, which is suggested by several realistic SUSY GUT models utilizing some discrete symmetries to realize the doublet-triplet splitting in SUSY GUTs [12]. The resultant baryon asymmetry is totally independent of the reheating temperature of inflation, and the required initial amplitude of the AD field is perfectly consistent with the $B-L$ breaking scale $v_{B-L} \approx M_{\mathrm{GUT}}$ suggested from neutrino-oscillation data. Furthermore, the abundance of thermal gravitino dark matter is also independent of the reheating temperature in a relatively wide parameter space. In that region, the abundance of gravitino dark matter is 
solely determined by the electroweak-scale parameters and the initial amplitude of the AD field. Encouragingly, the required amplitude of the AD field is quite consistent with the value required to explain the observed baryon asymmetry.

We have also discussed the nonthermal gravitino production via the late-time decays of Q-balls. The constraints from $\mathrm{BBN}$ and the observation of the large-scale structures in the Universe can be easily satisfied unless the messenger scale is too high, $M_{m} \geqslant 10^{10} \mathrm{GeV}$, or the gravitino is relatively heavy, $m_{3 / 2} \gtrsim 10 \mathrm{MeV}$. In the case of the $B$-ino NLSP, the "hotter" warm dark matter supplied by the nonthermal gravitino production is expected to be larger than a few percent of the total mass density of dark matter, whose existence may be tested by MAP or Planck experiments in the near future [34].

Finally, we add a comment on the effects of possible nonrenormalizable operators in the superpotential. The discrete symmetry, which gives us a natural solution to the doublettriplet splitting problem, may allow nonrenormalizable operators in the superpotential, which lift the relevant flat direction. For example, $Z_{4 R}$ symmetry [35] allow nonrenormalizable operators proportional to $\phi^{6}$, such as $\propto(\bar{U} \bar{D} \bar{D})^{2}$. In the presence of the gauged $\mathrm{U}(1)_{B-L}$ symmetry, the operator is written as $\delta W=\left(\lambda X / M_{*}^{4}\right) \phi^{6}$, where $\lambda$ is a coupling constant, and $X$ is the Higgs field carrying $B-L$ charge 2 to break the $B-L$ symmetry spontaneously. ${ }^{12}$ This operator lifts the $\phi$ field at $|\phi| \approx M_{*}\left(m_{x} / \lambda\langle X\rangle\right)^{1 / 4}$, which is of the same order as the $(B-L)$-breaking scale. Hence, even if this operator exists, it is still possible for the $\phi$ field to have its initial amplitude of the order of the $(B-L)$-breaking scale. The contribution of this operator to the baryon asymmetry is approximately given by $\left(n_{B} / n_{\phi}\right) \simeq\left(m_{3 / 2} / m_{x}\right) \delta_{\text {eff }}$, which is subdominant as long as $m_{3 / 2} \lesssim 1 \mathrm{MeV}$ respect to that in Eq. (4) [see also the discussion just below Eq. (30)]. Therefore, the results of the present model are basically independent of the existence of such nonrenormalizable operators in the superpotential.

\section{ACKNOWLEDGMENTS}

M.F. thanks the Japan Society for the Promotion of Science for financial support. This work was partially supported by Grant-in-Aid for Scientific Research (S) 14102004 (T.Y.).

\footnotetext{
${ }^{12}$ Assigning the $B-L$ charge 1 to the $X$ field and adopting a superpotential $\delta W=\left(\lambda X^{2} / M_{*}^{5}\right) \phi^{6}$ do not change the main arguments in the following discussion.
}

[1] M. Dine, A.E. Nelson, and Y. Shirman, Phys. Rev. D 51, 1362 (1995); M. Dine, A.E. Nelson, Y. Nir, and Y. Shirman, ibid. 53, 2658 (1996). For a review, see, for example, G.F. Giudice and R. Rattazzi, Phys. Rep. 322, 419 (1999), and references therein.

[2] T. Moroi, H. Murayama, and M. Yamaguchi, Phys. Lett. B 303, 289 (1993); A. de Gouvea, T. Moroi, and H. Murayama, Phys. Rev. D 56, 1281 (1997).

[3] I. Affleck and M. Dine, Nucl. Phys. B249, 361 (1985).

[4] G. Lazarides and Q. Shafi, Phys. Lett. B 258, 305 (1991); K. Kumekawa, T. Moroi, and T. Yanagida, Prog. Theor. Phys. 92, 437 (1994); G. Lazarides, Springer Tracts Mod. Phys. 163, 227 (2000), and references therein; G.F. Giudice, M. Peloso, A. Riotto, and I. Tkachev, J. High Energy Phys. 08, 014 (1999); T. Asaka, K. Hamaguchi, M. Kawasaki, and T. Yanagida, Phys. Lett. B 464, 12 (1999); Phys. Rev. D 61, 083512 (2000); M. Fujii, K. Hamaguchi, and T. Yanagida, ibid. 65, 115012 (2002); T. Asaka, H.B. Nielsen, and Y. Takanishi, hep-ph/0207023.

[5] K. Hamaguchi, H. Murayama, and T. Yanagida, Phys. Rev. D 65, 043512 (2002).

[6] S.R. Coleman, Nucl. Phys. B262, 263 (1985); B269, 744(E) (1986).

[7] A. Kusenko and M.E. Shaposhnikov, Phys. Lett. B 418, 46 (1998); M. Laine and M.E. Shaposhnikov, Nucl. Phys. B532, 376 (1998).

[8] S. Kasuya and M. Kawasaki, Phys. Rev. D 62, 023512 (2000); 64, 123515 (2001)

[9] H. Murayama and T. Yanagida, Phys. Lett. B 322, 349 (1994).

[10] T. Asaka, M. Fujii, K. Hamaguchi, and T. Yanagida, Phys. Rev. D 62, 123514 (2000); M. Fujii, K. Hamaguchi, and T. Yanagida, ibid. 63, 123513 (2001); 65, 043511 (2002).
[11] M. Fujii, K. Hamaguchi, and T. Yanagida, Phys. Lett. B 538, 107 (2002).

[12] R. Barbieri, G.R. Dvali, and A. Strumia, Phys. Lett. B 333, 79 (1994); K.I. Izawa and T. Yanagida, Prog. Theor. Phys. 99, 423 (1998); K. Kurosawa, N. Maru, and T. Yanagida, Phys. Lett. B 512, 203 (2001); E. Witten, hep-ph/0201018.

[13] T. Yanagida, Phys. Lett. B 400, 109 (1997).

[14] M. Fujii and K. Hamaguchi, Phys. Rev. D 66, 083501 (2002).

[15] M. Fujii and T. Yanagida, Phys. Lett. B 542, 80 (2002).

[16] R. Allahverdi, A. Mazumdar, and A. Ozpineci, Phys. Rev. D 65, 125003 (2002).

[17] M. Dine, L. Randall, and S. Thomas, Phys. Rev. Lett. 75, 398 (1995); Nucl. Phys. B458, 291 (1996).

[18] M. Fujii, K. Hamaguchi, and T. Yanagida, Phys. Rev. D 64, 123526 (2001).

[19] R. Banerjee and K. Jedamzik, Phys. Lett. B 484, 278 (2000).

[20] A.G. Cohen, S.R. Coleman, H. Georgi, and A. Manohar, Nucl. Phys. B272, 301 (1986).

[21] K. Enqvist and J. McDonald, Nucl. Phys. B538, 321 (1999).

[22] T. Yanagida, in Proceedings of the Workshop on the Unified Theory and the Baryon Number in the Universe, Tsukuba, Japan, 1979, edited by O. Sawada and A. Sugamoto (KEK Report No. KEK-79-18, Tsukuba, 1979), p. 95; Prog. Theor. Phys. 64, 1103 (1980); M. Gell-Mann, P. Ramond, and R. Slansky, in Supergravity, edited by D. Z. Freedman and P. van Nieuwenhuizen (North-Holland, Amsterdam, 1979).

[23] T. Moroi and T. Takahashi, Phys. Lett. B 522, 215 (2001); 539, 303(E) (2002); Phys. Rev. D 66, 063501 (2002). See also D.H. Lyth and D. Wands, Phys. Lett. B 524, 5 (2002).

[24] See, for example, T. Asaka, K. Hamaguchi, M. Kawasaki, and T. Yanagida, in Ref. [4] and references therein. 
[25] MAP webpage, http://map.gsfc.nasa.gov.

[26] Planck webpage, http://astro.estec.esa.nl/SA-general/Projects/ Planck.

[27] de Gouvea, Moroi, and Murayama [2].

[28] M.Y. Khlopov and A.D. Linde, Phys. Lett. 138B, 265 (1984); J.R. Ellis, J.E. Kim, and D.V. Nanopoulos, ibid. 145B, 181 (1984); M. Kawasaki and T. Moroi, Prog. Theor. Phys. 93, 879 (1995); M. Kawasaki, K. Kohri, and T. Moroi, Phys. Rev. D 63, 103502 (2001); K. Kohri, ibid. 64, 043515 (2001).

[29] M. Fujii and K. Hamaguchi, Phys. Lett. B 525, 143 (2002).

[30] J.R. Ellis, G.B. Gelmini, J.L. Lopez, D.V. Nanopoulos, and S.
Sarkar, Nucl. Phys. B373, 399 (1992).

[31] V.K. Narayanan, D.N. Spergel, R. Dave, and C.P. Ma, astro-ph/0005095.

[32] W.B. Lin, D.H. Huang, X. Zhang, and R.H. Brandenberger, Phys. Rev. Lett. 86, 954 (2001).

[33] Xiaomin Wang, M. Tegmark, and M. Zaldarriaga, Phys. Rev. D 65, 123001 (2002).

[34] D.J. Eisenstein, W. Hu, and M. Tegmark, Astrophys. J. 518, 2 (1998).

[35] Kurosawa, Maru, and Yanagida [12]. 